\title{
Reply to: Comment on Huang et al.: One-stage surgical management for children with spinal tuberculosis by anterior decompression and posterior instrumentation
}

\author{
Changkun Zheng
}

Received: 27 January 2010 / Published online: 25 February 2010

(C) Springer-Verlag 2010

\section{Dear Editor,}

We read the comments by Jain Saurabh [1] concerning our article [2]. Thank you for the comments. Conservative treatment with antituberculosis drugs and external immobilisation is the first choice. Aggressive surgical treatment should be adopted in the management of indicated patients with spinal tuberculosis. We treated more than 15 patients conservatively. Only 15 indicated patients failed to respond to the conservative treatment. Indications for surgery are neurological deficits, spinal instability, severe and progressive kyphosis, no response to chemotherapeutic treatment, non-diagnostic biopsy and large paraspinal abscess. Despite effective treatment for spinal tuberculosis, it is common to find residual kyphosis and instability at the end of treatment. In our 15 patients this resulted in an improvement in the correction of the deformity. At follow-up, progression of kyphosis was seen. The posterior internal fixation through the posterior column of the spine keeps the internal fixation away from the tuberculous focus, which is beneficial for tubercular resolution. The term resection/debridement should not be interchanged. The aim of this clinical study was to assess the efficacy of one-stage surgical management for children with spinal tuberculosis by anterior decompression, bone grafting, posterior instrumentation, and fusion; but there are too few indicated cases to resolve the issue.

\section{References}

1. Jain S (2009) Comment on : Qi shan Huang et al on one stage surgical management for children with spinal tuberculosis by anterior decompression and posterior instrumentation. Int Orthop. 25-Nov-2009

2. Huang Q-S, Zheng C, Hu Y, Yin X, Xu H, Zhang G, Wang Q (2009) One-stage surgical management for children with spinal tuberculosis by anterior decompression and posterior instrumentation. Int Orthop 33:1385-1390

\section{Zheng $(\square)$}

Department of Spine Surgery,

$\mathrm{Pu}$ Ai Hospital of Tongji Medical College of Huazhong

University of Science and Technology,

Wuhan, People's Republic of China

e-mail: zck2003@163.com 